\title{
Innovación en la organización de proyectos de Educación Continua mediante un modelo cuántico
}

\section{Innovation in the organization of education continuing projects by using a quantum model}

\author{
Manuel Campos-Rudin ${ }^{1}$ \\ Universidad de Costa Rica, Costa Rica
}

\begin{abstract}
Resumen. Los programas de Educación Continua (educación a lo largo de la vida) gestados dentro de las universidades de forma tradicional optan por el llamado modelo newtoniano. Este modelo se ajusta a los estilos occidentales de gestión y manejo de las organizaciones, pero parece ser insuficiente para las demandas de conocimiento que requiere la sociedad en las puertas de la llamada cuarta revolución industrial. Un modelo cuántico puede ser una forma innovadora de gestar programas y proyectos de educación continua que se adapten mejor a los requerimientos de nuestra sociedad, y las universidades pueden aprovechar de manera óptima tal modelo. El presente estudio cumple como objetivo mostrar los beneficios que se obtienen al plantear un esquema cuántico sobre uno newtoniano en Educación Continua. Se compararon dos programas de educación continua de dos unidades académicas diferentes por un periodo similar de cuatro años. Los resultados demostraron que el modelo cuántico permite optimizar el desempeño de los programas al facilitar procesos flexibles en su gestión, empoderar a los actores y gestar una organización que responde a las demandas de forma integral. Adicionalmente, permitió la transición de la coordinación; incluyó la presencia activa de profesores de otros países y la participación de estudiantes de posgrado; propició proyectos de acción social y logró sostenibilidad y crecimiento continuo por más de cuatro años. Se concluye que el proceso de innovación basado en un modelo cuántico, por sus características innatas, permite una mejor capacidad organizacional sobre el modelo newtoniano.
\end{abstract}

Palabras clave. Aprendizaje continuo a lo largo de la vida, gestión, modelo cuántico, innovación organizacional, Universidad de Costa Rica.

\begin{abstract}
Universities' continuing education (long life education) programs managed traditionally uses the Newtonian Model. This model adjusts to the western style of organizations management, but it seems insufficient due to the knowledge demand required by current society at the fourth industrial revolution. A Quantum Model may be an innovative way to manage programs and projects of continuing education that adapt better to our society requirements and improve the knowledge transmission of high investigative universities and schools. The aim of this study is to show the benefits obtained with a Quantum outline over a Newtonian one in continuing education. During a similar time (4 years), two continuing education programs from two different schools were compared. Results showed that the Quantum Model optimizes programs performance because it facilitates processes flexibility in their management, it empowers persons involved, and it allows managing an organization that responds to current demands integrally. Also, this model permits the transition of the coordination, including the active presence of foreign professors, the integration of postgraduate students favoring social projects and achieving sustainability with continuing improvement for more than four years. To conclude, this innovative process based on the Quantum Model, due to its inherent traits, allows a better organizational capacity in comparison with the Newtonian Model.
\end{abstract}

Keywords. Lifelong Learning, Management, Quantum Model, Organizational Innovation, University of Costa Rica. 


\section{Introducción}

La Educación Continua (EC) es "una modalidad educativa complementaria al sistema formal dirigido a la formación integral de las personas a lo largo de toda la vida" (López, 2013, p. 51). Los términos EC y aprendizaje continuo a lo largo de la vida se consideran sinónimos. Estos procesos brindan la oportunidad a las personas de transmitir conocimientos e incrementar su calidad de vida; además, es una herramienta para que las organizaciones sean exitosas (Laal, Laal, \& Aliramaei, 2014).

Las personas egresadas de las universidades requieren procesos en EC para alimentar el flujo de conocimientos de la academia al entorno que las rodea (sociedad y país). La EC les permite a estas poblaciones mantener vigentes sus conocimientos, certificarlos por una vía no formal, concentrarse en aquellos temas que demandan su atención (no necesariamente de su formación académica básica) y lograr mediar ante toda la sociedad para tener acceso a los conocimientos emanados de las universidades (Velazco, 2011). Esto muestra que una de las muchas posibles herramientas de que dispone la sociedad para mejorar la condición de sus pueblos y naciones es lograr un proceso educativo y de aprendizaje continuo durante la vida de sus integrantes, por lo que se vuelve una actividad que no acaba (Tuschling \& Engemann, 2006).

En nuestros días, la EC se convierte en una herramienta para que profesionales $\mathrm{o}$ tecnólogos egresados de unidades académicas con alto desempeño en investigación (UAI) puedan mantener vigentes y actualizados sus conocimientos para el bien de la sociedad donde laboran y conviven. Las UAI proceden de forma regular a impulsar proyectos de investigación y gestar cursos regulares a sus estudiantes. Pero en muchas escuelas del tipo UAI centran sus esfuerzos en la investigación y, luego, en educación formativa y no incorporan como una parte integral de su acción la EC (Anderson, Banerjee, Elgin, \& Handelsmann, 2011). Incluso, en universidades con énfasis en investigación, el sistema de premiación docente se basa en la investigación reflejada en la cantidad de artículos científicos publicados, la docencia parece ser no recompensada de la misma forma y hasta el tiempo dedicado a esta última puede ser afectado pues el grupo docente debe emplearse a fondo en la búsqueda de recursos económicos para las investigaciones, $\mathrm{y}$, por último, se presenta la EC dentro de la acción social de las universidades.

Ante el panorama anterior, la EC en las UAI debe dinamizarse a las puertas de la llamada cuarta revolución industrial, la cual es impulsada en gran medida por el amplio desarrollo de las tecnologías como: nanotecnología, biotecnología, neurociencias, robótica einteligencia artificial. En síntesis, podemos decir que la cuarta revolución industrial:

no solo consiste en máquinas y sistemas inteligentes y conectados. Su alcance es más amplio. Al mismo tiempo, se producen oleadas de más avances en ámbitos que van desde la secuenciación genética hasta la nanotecnología, y de las energías renovables a la computación cuántica. Es la fusión de estas tecnologías y su interacción a través de los dominios físicos, digitales y biológicos lo que hace que la cuarta revolución industrial sea fundamentalmente diferente de las anteriores (Schwab, 2016, s.p.).

En su conjunto, la cuarta revolución industrial fomenta que la información y el intercambio de conocimientos ocurran en tiempo real y su interacción promueve que estas sean casi imposibles de seguir por las personas no vinculadas a las UAI. Por el contrario, los profesionales sí vinculados a las UAI adquieren facilidades para asistir a congresos, simposios, años sabáticos, posgrados o post-doc, así como permisos para realizar pasantías con goce de salario en otros países a fin de compartir experiencias y especializarse. 
Desde este precepto, disciplinas como ciencias biomédicas, biotecnología, ciencias biológicas (ecología, botánica, zoología, embriología, fisiología, ciencias marinas y manejo de recursos naturales, por citar algunas) y otras disciplinas afines como agronomía, bioprocesos e ingeniería de biosistemas se hallan inmersas en este flujo intenso de conocimientos entre ellas. $Y$ dichos procesos deben unirse o fusionarse a los de otras ingenierías que tradicionalmente no se vinculaban con temas biológicos; este es un hecho imparable. Si un país desea un desarrollo y crecimiento sostenible debe generar espacios para el intercambio de pensamientos con la población en general. La innovación en nuestros tiempos debe ser parte integral de cualquier organización y los procesos para gestarla ya han sido previamente descritos (de Bes \& Kotler, 2011).

Lo anterior induce a pensar que la formación académica universitaria y la EC deben migrar hacia un proceso de innovación continua, puesto que, de otra forma, no podrán alinearse a las demandas de la población; deben, así, generarse nuevos modelos para queinteractúen estudiantes y profesores (Pehlivanova \& Ducheva, 2010). De manera óptima, la EC puede desarrollarse como una organización cuántica e innovadora que le permita ajustarse rápidamente a este nuevo modelo en el cual lo biológico se funde con lo tecnológico o material.

El objetivo del presente estudio es mostrar la experiencia y los resultados de dos Programas de Educación Continua (PEC) en unidades académicas de tipo UAI de la Universidad de Costa Rica (UCR). Una de ellas gestada mediante una estructura organizativa tipo newtoniana en la Escuela de Medicina (2000-2003) y otra que se gestó de forma innovadora bajo un modelo cuántico en la Escuela de Biología (2010- 2013 y que permanece activo hasta la fecha de presentar este artículo). Se pretende mostrar cómo un modelo cuántico es una alternativa por considerar para los PEC de las UAI en la era de la cuarta revolución industrial.

\section{Marco teórico}

En nuestro mundo occidental, el modelo de organización newtoniana tiende a predominar; así pues, de forma reciente, modelos cuánticos son mencionados como la alternativa viable para generar un crecimiento continuo y sostenible. Las organizaciones newtonianas presentan controles rígidos y estructurados entre sus características básicas, con el fin de lograr un proceso eficiente de producción. El modelo newtoniano se basa en doctrinas y posiciones jerárquicas que buscan obtener metas y resultados; y cuando se forman unidades de acción o de trabajo, estos se enfocan en sus tareas sin lograr comunicarse entre ellas (Zohar, 2016). Tales esquemas son rígidos y les dificultan a las organizaciones responder o adaptarse de forma rápida ante cambios en su ecosistema pues no inducen la generación de procesos innovadores y si estos ocurren deben fluir a través de canales poco flexibles y de bajo empoderamiento. Estas organizaciones con modelos newtonianos tienen dificultad para realizar procesos de aprendizaje que generen una transformación continua (Yavas \& Polat, 2015).

En otras palabras un modelo newtoniano explota las virtudes de un gran líder o "gerente" que controla y dirige la organización, donde además es difícil cuestionar su autoridad o realizar procesos innovadores sin que estos no sean avalados de forma previa por este "gerente" o líder, y la organización responderá a cambios únicamente si esta persona así lo desea (tabla 1). En la figura 1 se presenta un ejemplo del modelo newtoniano (MN) adaptado a un director de una Escuela Académica, donde este tiene bajo su dirección la jefatura administrativa y en el otro extremo la dirección de los diferentes coordinadores de secciones o departamentos; 
cada de una de estas unidades representa un colaborador que opera bajo una serie de lineamientos estrictamente definidos. Las líneas sólidas de la figura 1 indican claramente una estructura de mando no flexible, sin empoderamiento para los actores. En este MN se observa cómo la interacción o comunicación horizontal es restringida y las experiencias obtenidas por una unidad no tienen que ser conocidas o comunicadas a otra unidad.

Las organizaciones cuánticas presentan varias características, de las que podemos señalar: autoconciencia gerencial flexible, presencia de actores internos activos (directores, coordinadores, docentes y administrativos), se busca promover el empoderamiento y una meta

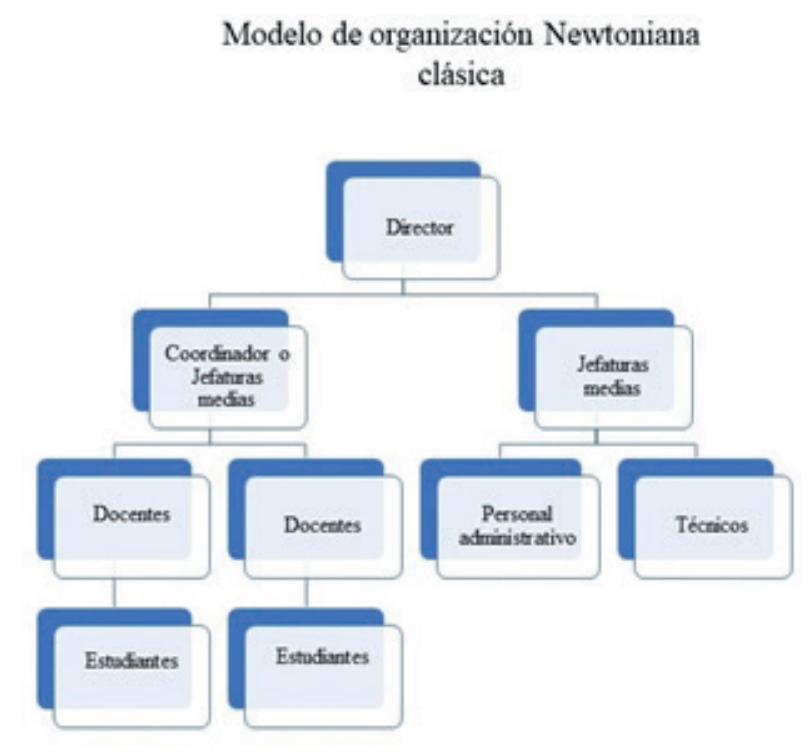

es el eterno proceso de transformación (Kilmann, 2011). En la figura 1 se presenta un modelo de organización cuántica. Se puede notar que esta no busca crear patrones jerárquicos rígidos y esta proyecta flexibilidad. En la tabla 1 se detallan las características del modelo cuántico (MC). El MC nos hace pensar y cuestionar si nuestros patrones o modelos actuales deben migrar hacia nuevas formas, pues en las últimas décadas es claro que los procesos formales y rígidos educativos no son la solución para los retos que nuestra sociedad afronta para crecer y ser sostenible en el tiempo (Severin, 2017). En la tabla 1 podemos contraponer las diferencias de las siete características de la organización cuántica y su contraparte newtoniana:
Modelo de organización Cuántica



Figura 1. Modelos básicos de una organización newtoniana y una cuántica. Fuente. Campos-Rudin (2018) 
Tabla 1

Características básicas de las organizaciones newtonianas y cuánticas

\begin{tabular}{|c|c|}
\hline Modelo newtoniano & Modelo cuántico \\
\hline $\begin{array}{l}\text { MN1. La conciencia es excluida del diseño formal de la } \\
\text { organización y su guía es gerencial. }\end{array}$ & $\begin{array}{l}\text { MC1. La conciencia está incluida dentro del diseño } \\
\text { y su guía es con base en liderazgo. }\end{array}$ \\
\hline $\begin{array}{l}\text { MN2. La organización es simple y está compuesta por } \\
\text { trabajadores que siguen procedimientos oficiales. Se bus- } \\
\text { ca que sea predecible. }\end{array}$ & $\begin{array}{l}\text { MC2. La organización es compleja, pero los partici- } \\
\text { pantes están activamente incluidos dentro del dise- } \\
\text { ño de la organización. No es predecible. }\end{array}$ \\
\hline $\begin{array}{l}\text { MN3. Los trabajadores pasivos son ignorados y trabajan } \\
\text { de forma independiente. }\end{array}$ & MC3. Se rompen fronteras por los participantes. \\
\hline $\begin{array}{l}\text { MN4. La administración no está consciente de que están } \\
\text { en una organización rígida y reactiva. }\end{array}$ & $\begin{array}{l}\text { MC4. La organización es conducida de forma flexi- } \\
\text { ble. Hay imaginación y experimentación. }\end{array}$ \\
\hline MN5. Controles externos a los trabajadores. & $\begin{array}{l}\text { MC5. Participación de los integrantes de la orga- } \\
\text { nización en forma activa. Se fomenta la integra- } \\
\text { ción-comunicación. }\end{array}$ \\
\hline $\begin{array}{l}\text { MN6. Segregación forzada de los trabajadores pasivos } \\
\text { (fragmentación). No se propicia empoderamiento. }\end{array}$ & MC6. Fuerte empoderamiento de los participantes. \\
\hline $\begin{array}{l}\text { MN7. La estructura rígida de la organización lleva a un } \\
\text { proceso autodestructivo. }\end{array}$ & $\begin{array}{l}\text { MC7. Una organización flexible que se transforma } \\
\text { de manera continua (eterna). }\end{array}$ \\
\hline
\end{tabular}

Fuente. Adaptado de Kilmann, R., 2011., pp. 66-69

De la información presentada en la tabla 1, se puede deducir que un proceso educativo debe considerar evolucionar y crecer hacia una "intromisión cuántica", un término planteado por Giraldo (2017). En América Latina, se debe pensar seriamente en tomar acciones para aplicar procesos cuánticos en la educación para así no quedar rezagados ante la revolución de conocimientos que están presentes en nuestros días (Giraldo, 2017). Pero esto no implica perder la rigurosidad científica y no asegurar un control de calidad sobre las propuestas educativas; por lo tanto, un MC puede acoplar algunas estructuras Newtonianas para lograr un óptimo desempeño educativo (Jones, 2017) .

Procedemos ahora a una síntesis conceptual: un PEC busca educar a las personas a lo largo de la vida. Es contradictoria la creación y gestión de un PEC que al ejecutar un MN puro lleve a gestar un proceso autodestructivo o ser tan rígidos que no pueden evolucionar (reflejado en la tabla 1 en el punto MN7). Si los seres humanos requerimos evolucionar en nuestros conocimientos en la cuarta revolución industrial, los PEC deben adquirir elementos de los MC para cumplir su objetivo principal: educar a lo largo de la vida. Este puede ser el mejor indicador de un éxito PEC: ser una organización que pueda seguir un MC de transformación continua, como se postula en el MC7 de la tabla 1.

A partir de la premisa de que un PEC requiere evolucionar y transformarse en forma continua, el MC parece ser un proceso que propicia la innovación al ser integrador (Zohar, 2016). La innovación puede ser definida como proceso de aprendizaje continuo, investigación $y$ 
exploración, que refleja una serie de mejoras en la producción, nuevos productos o servicios, cambios en las organizaciones y búsqueda de nuevos mercados y todo lo que realizamos trae como consecuencia mejoras en los servicios y procesos. Esta innovación puede estar vinculada a las organizaciones (como un PEC) donde habrá cambios en: relaciones de autoridad, estructura organizacional, tipos de trabajos, sistemas de remuneración económica y cambios en como las personas o actores de la organización interaccionen entre ellos mismos (Marques, 2014). Se puede resumir que la innovación busca realizar cambios tecnológicos y en el modelo del servicio que se oferta (modelo de negocios) y la EC es parte integral de una innovación sostenible.

Se proponen dos tipos de invocación: la incremental y radical. La incremental es la innovación que realizamos de forma cotidiana, nos permite mejorar lo que hacemos diariamente, son de bajo riesgo y de forma predecible busca hacernos más productivos. En la innovación radical, se toman grandes riesgos para lograr éxito; puede ser al usar tecnologías disruptivas en el mercado o al cambiar el modelo de negocios de forma contundente. Entre estos dos tipos de innovación, podemos encontrar un grupo intermedio: la semi-radical con dos alternativas, i) la semi-radical, en que solo hay cambios tecnológicos, aunque no se gestiona un nuevo modelo de negocios y ii) la semi-radical, en que no hay cambios tecnológicos, pero sí cambios en el modelo de negocios o de la organización (Dávila, 2008). En un MN, se pueden desarrollar innovaciones incrementales con alta facilidad; pero las radicales, por su estructura rígida, tomarán más tiempo. En el $\mathrm{MC}$, es posible encontrar que las organizaciones se hallan más preparadas para adoptar innovaciones semiradicales o radicales, que favorezcan cambios tecnológicos disruptivos y nuevos modelos de negocios o servicios.

\section{Metodología}

En dos escenarios distintos en tiempo y espacio, se gestaron dos PEC en dos escuelas diferentes de la UCR. Las dos únicas similitudes fueron la participación del mismo coordinador y el deseo de los directores de ambas Escuelas para establecer PEC funcionales como parte de su visión directiva. Con el objetivo de realizar una comparación efectiva, se analizarán los cuatro primerosañosdeoperación deambosPEC, tiempo necesario para formar y desarrollar un programa capaz de mostrar resultados consistentes

PEC de la Escuela de Medicina

El PEC-Escuela de Medicina (EM) fue gestado en el 2000 y permaneció vigente hasta 2004; a finales de ese año no se renovó su gestión y se le dejó la responsabilidad al PEC de la Facultad de Medicina (la cual incluye varias Escuelas de ciencias médicas o afines), con el fin de no duplicar esfuerzos hacia un mercado o población meta similar.

Este PEC de la EM surgió en el entorno médico del 2000, cuando el Colegio de Médicos y Cirujanos de Costa Rica buscaba que sus agremiados certificaran su actualización profesional. En aquel contexto, la dirección de la EM buscó desarrollar un PEC como entidad acreditada para dar cursos de actualización a los médicos. Se desarrolló un modelo de innovación incremental al buscar mejorar los procesos conforme el coordinador del PEC acumulaba experiencias de años anteriores, como reducir el tiempo para ejecutar una actividad, ahorrar recursos económicos en la divulgación de actividades y reaccionar de forma oportuna ante unanecesidad delmercadoentemasdeeducación.

El PEC-EM se gestó mediante un MN (similar a la gran mayoría de estructuras docentes de la universidad) y se aplicaron los lineamientos explicados en la tabla 1: 
MN1. La conciencia es excluida del diseño formal de la organización y su guía es gerencial. El director de la EM daba las instrucciones de forma puntual a sus mandos medios o jefaturas, se presentaban informes operativos y era difícil coordinar entre los diversos departamentos y con otras jefaturas.

MN2. La organización es simple y está compuesta por trabajadores que siguen procedimientos oficiales. Se busca que sea predecible. La temática de los cursos fue coordinada y gestada de forma directa entre el director de la EM y el coordinador del programa. Si se dio una convocatoria a docentes de la EM para presentar posibles ideas de cursos o talleres, estas no dieron resultados positivos y en general no se observó mucho interés por parte del cuerpo docente en participar. Los docentes, en muchos casos, eran contratados y se les pagaba un honorario establecido por la coordinación del PEC (avalado por la dirección de la EM).

MN3. Los trabajadores pasivos son ignorados y trabajan de forma independiente. La EM no aportó ningún recurso económico para el PEC-EM, salvo el tiempo docente de coordinador del PEC; los demás gastos debían cubrirse por los ingresos mismos que generaba la matrícula de los cursos. El docente coordinador del PEC, para maximizar su gestión, fue asignado únicamente a labores dentro del programa, sin vinculación con docencia ni investigación en la EM y sus respectivos departamentos; se le asignó un tiempo de 30 horas semanales para desarrollar el proceso. En el segundo año, se dedicó 10 horas semanales y se asignó un profesional adicional con medio tiempo para labores administrativas. En su momento, la dirección consideró que el coordinador del PEC debía estar únicamente vinculado a la operación del programa, tampoco se vinculó al coordinador para que trabajara de forma coordinada con la sección administrativa. Era un programa aislado dentro de la EM.
MN4. La administración no está consciente de que están en una organización rígida y reactiva. Se impartían cursos presenciales de 10 a 40 horas de duración máxima. Se brindaron clases presenciales y el grupo de profesores estaba conformado por colaboradores de la EM y exclusivamente en casos de congresos o visita de algún investigador de otra universidad, había un cambio en el perfil de las actividades. Con respecto a docentes distintos a la EM o extranjeros, el PEC-EM no realizó gestiones para invitarlos al país, sino que fue más por efectos colaterales de su visita a la UCR que se logró su inserción en cursos de capacitación del PEC. No se desarrollaron capacitaciones dirigidas a empresas. El coordinador del PEC de la EM no pudo operar diferentes modelos educativos.

Análisis de los resultados e informes obtenidos en las actividades por PEC-EM

La población meta eran profesionales en ciencias afines a la medicina $y$ no se contemplaron cursos para personas no graduadas de universidades o con grados técnicos o empíricos, ni se buscó capacitar profesionales de otras disciplinas distintas a la biomédica.

Al finalizar cada curso, se aplicó una encuesta de salida a los participantes para evaluar si la formación cumplió sus expectativas, si el docente ofreció de forma efectiva el temario, si las aulas eran apropiadas, si el material audiovisual era adecuado y si había observaciones adicionales. Dicho instrumento de medición está disponible en la Vicerrectoría de Acción Social (VAS) de la UCR para su empleo en todos los eventos de capacitación. Respecto de cada evento, debía presentarse un informe de impacto académico e impacto social, así como datos de ingresos y gastos generados. Al finalizar cada año de operación, se elaboraba un informe completo a la dirección de la EM y a la VAS. 
Los indicadores de medición del PEC de la EM fueron: cantidad de cursos (al menos debía gestarse entre ocho y diez actividades por año) y si el programa era sostenible económicamente pues no contaba con presupuesto de la UCR para realizar ninguna actividad. No existió retroalimentación de otros docentes evaluadores por parte de la EM.

Programa de Educación Continua y Asesoramiento en Ciencias Biológicas (PECACIBI) de la Escuela de Biología

La Escuela de Biología (EB), antes del 2010, desarrolló un proyecto de EC denominado Biología para Todos. Posterior al 2010, se definió por la dirección de la EB replantear un PEC que retome los logros anteriores y gestar un proceso de innovación.

Como primer paso, se revisaron la misión y la visión de la EB para determinar el rol de la acción social y su efecto en la EB. Se determinó que la EC sí estaba incluida, pero se observó que en la organización y gestión de la EB existía (y aún) un fuerte predominio de la investigación y docencia, lo cual la posicionaba como una UAI. De esta forma, se determinó que el lanzamiento de un nuevo PEC requería un proceso innovador para incentivar a los docentes a participar. Como activador de la innovación se realizó un modelo de activación -In out- donde, desde la dirección y coordinación del PEC se dirigió el proceso hacia la parte científica-docente (de Bes \& Kotler, 2011).

Para inducir un proceso innovador, el coordinador del PEC buscó gestar un MC en la EB. El MC plasmado por PECACIBI innovó al utilizar, en su conceptualización y operación, varios de los elementos cuánticos descritos en la tabla 1:

MC1. La organización es conducida de forma flexible. Hay imaginación y experimentación. A propósito de este enunciado, el director de la
EB pudo sugerir o plantear posibles cursos o actividades; pero no impuso un estricto orden de operaciones. Dejó al coordinador con la flexibilidad operativa necesaria para interactuar de forma individual con los docentes de la EB, en aras de motivarlos a realizar procesos de EC. La "gerencia", en este caso, fue compartida entre coordinador del PEC y el director de la EB. También se ofrecieron opciones para que los docentes definieran sus temas, sus intereses y la forma en que los excedentes generados por los ingresos por concepto de matrícula podían emplearse. Así, una parte de estos recursos iba a la EB para su gestión operativa; y de la otra parte, el profesor o grupo docente podía solicitar un pago por honorarios o compra de reactivos o insumos para su gestión universitaria.

Pero sí se debe aclarar que, para el caso de honorarios profesionales, el PEC-EB, en su formulación estableció una hora profesional similar y competitiva con los parámetros estipulados por el Colegio de Biólogos de Costa Rica, por el precepto de que cada evento debía ser sostenible y si la población meta del curso requería en forma parcial o total becas, se buscarían los mecanismos para darlas. Para conocer más detalles sobre los lineamientos de pagos y administración de excedentes, revisar Campos-Rudin (2017).

No se buscó un modelo rígido para cada evento, sino ser flexibles para lograr una gestión eficiente de todo el PEC. La flexibilidad no cumplió como objetivo obtener más ingresos o rentabilidad, sino ser efectivos en la conducción del PEC. Un ejemplo del proceso de flexibilidad y experimentación fue un curso de educación continua que se realizó en la Isla de Chira (provincia de Puntarenas), dirigido a pescadores artesanales.

MC2. La organización es compleja, pero los participantes están activamente incluidos dentro del diseño de la organización (no es predecible). Se 
determinó la existencia de cuatro participantes internos: los docentes involucrados en el curso, el personal de la sección administrativa de la EB, el coordinador del PEC y el director de la EB. El docente, de manera usual, no fue contratado o solicitado en un evento para que impartiera una charla impuesta o un curso con un programa de objetivos rígido y pre-estructurado desde la dirección y coordinación del PEC. Además, el docente tuvo la libertad para actuar de forma activa durante el evento.

El personal administrativo era informado con anticipación del evento y se le brindaban datos e informes de los objetivos y demandas del curso en términos de aulas, laboratorios y requerimientos para la puesta en marcha. Así, la jefatura de la sección administrativa pudo asesorarse sobre si las fechas y recursos estaban disponibles para la actividad. El coordinador del PEC sirvió de enlace y facilitador entre todos los participantes activos; y el director, en caso de ser necesario, tomaba alguna posición si no lograba un consenso en un punto. En resumen, cada curso tenía como modelo una interacción creativa de los docentes del curso, soporte del coordinador del PEC, y apoyo consensuado de la sección administrativa y supervisión activa, pero flexible por parte director de la EB.

MC6. Fuerte empoderamiento de los participantes. El grupo de docentes de cada curso estaba empoderado de forma total para determinar $100 \%$ de programa académico y las horas de acción definidas en la propuesta original. Sin embargo, siempre se buscó que el coordinador del PEC asesorara y orientara al docente en la confección del programa.

El PEC daba el empoderamiento para que los docentes pudieran divulgar el evento entre sus redes o invitar a expositores (no necesariamente profesores de la UCR), por ejemplo; también podían invitar a estudiantes de posgrado para impartir una charla e incluso se les permitía definir la intensidad y duración del curso. Este empoderamiento estaba ligado a una estrecha comunicación con los demás actores internos del curso, descritos en el apartado anterior.

\section{MC7. Una organización flexible que se transforma} de manera continua (eterna). Cada curso o evento siempre contó con una evaluación de los participantes y la presentación de los informes pertinentes solicitados por la VAS. Esos datos eran empleados para formular una respuesta rápida en mejora continua y el coordinador del PEC trabajó de forma directa con la comisión de acción social de la EB. Esta comisión evaluó los informes de cada evento y se le daba una retroalimentación al coordinador por parte de todos los miembros de comisión (todos profesores no vinculados de forma directa al PEC). La finalidad era lograr realizar mejoras o ajustes de forma continua en nuevos eventos del PEC. Tales recomendaciones abordaban aspectos, operativos, temáticos y resultados de sostenibilidad del PEC.

El MC implementado se complementó con la aplicación de elementos del MN, los cuales en su mayoría fueron incorporados para cumplir con la rigurosidad académica y científica que la UCR exige:

MN2. La organización es simple y está compuesta por trabajadores que siguen procedimientos oficiales. Se busca que sea predecible. Al ser un PEC inscrito oficialmente en la VAS, debió seguir procesos de oficiales de inscripción de eventos ante el comité de acción social de la EB, presentó reportes, elaboró informes parciales y finales (siguiendo los lineamientos institucionales), hubo emisión de certificados según normativas de la UCR y siguió los protocolos ya establecidos dentro de la Universidad para este tipo de actividades. Pero PECACIBI si flexibilizó su posición en (MC1) para evitar ser un $\mathrm{MN}$ típico en este aspecto y no ser predecible. 
MN5. Controles externos a los trabajadores. Todo evento debió ser evaluado por los participantes con una encuesta de salida, según el formulario que exige la VAS, el cual siempre fue solicitado a los participantes al terminar cada evento. La información era tabulada y analizada para su análisis. Adicionalmente, todos los que se capacitaron debieron firmar su asistencia y validar así la participación o, en su defecto, el aprovechamiento del curso si había exámenes.

El coordinador, de forma discreta, validaba las horas efectivas del curso y el desarrollo del evento, y actuaba como facilitador cuando hubo inconvenientes. Los informes que el coordinador presentaba eran revisados por profesores no vinculados al PEC, con apoyo de la comisión de acción social de la EB. Asimismo, el coordinador presentaba un plan de trabajo. En estos aspectos si se utilizó un MN completo, pero que no predominaran sombre el MC.

La gestión de PECACIBI buscó desarrollar ejes de articulación mucho mayores a los del proyecto de EC anterior, dirigiendo su acción a diversos públicos meta:biólogosy profesionalesegresados de las ciencias biológicas afines, investigadores, público en general, organizaciones privadas y públicas, así como brindar asesorías puntuales en temas biológicos. Su gestión y tipos de actividades pueden consultarse en CamposRudin, (2017). En breve, fue lo siguiente:

i. Cursos de capacitación de 10-40 horas con profesores de la Escuela de Biología.

ii. Cursos de capacitación de 10-40 horas con presencia de profesores invitados de otros países y de la Escuela de Biología.

iii. Cursos de capacitación de 10-40 horas con profesores de la Escuela de Biología y estudiantes de posgrado que colaboraron en el evento como expositores.

iv. Cursos de capacitación de 10 horas con investigadores de otros países en coordinación con otras unidades académicas o centros de investigación.

v. Cooperación con la gestión de simposios y congresos.

vi. Cursos especializados para biólogos o empresas privadas.

vii. Asesorías puntuales en temas biológicos al sector público o privado.

viii. Foros, coloquios o ponencias magistrales a docentes, investigadores y estudiantes de la Universidad de Costa Rica, y abiertos al público en general.

ix. Inducción a la gestión de proyectos de acción social con modalidad de extensión docente, dentro de su organización.

El tipo de innovación implementado fue de tipo semi-radical, al buscar mejorar y dinamizar el modelo de servicios en EC. Se desarrolló un $\mathrm{MC}$ con elementos newtonianos y no se realizaron cambios tecnológicos (como e-learning o educación virtual).

\section{Resultados}

\section{PEL PEC-EM y el MN empleado}

La EM posee diversos departamentos y directores por cada uno. En el área de ciencias básicas es posible citar Anatomía, Fisiología, Bioquímica y Farmacia, pero tiene muchos departamentos ubicados en los diversos hospitales del país, donde los estudiantes continúan su proceso formativo. El modelo organizativo del PEC de la EM se presenta en la figura 2. Desde la definición de organización newtoniana, se considera que el PEC-EM fue exitoso en los indicadores respectivos, planteados en su concepto:

i) Tuvo un inicio de cero pues no existía ningún proyecto de EC previo y logró ser sostenible por 4 años. 
ii) Pudo ofrecer cursos y actividades en un promedio de 10 por año después de su segundo año de operación y en el 2004, específicamente, realizó cuatro eventos.

iii) Fue financieramente sostenible y dejó excedentes a la EM para que fueran empleados en cubrir necesidades operativas.

iv) Canalizó capacitación al sector médico.

v) La innovación incremental en términos de mejoras en los procesos permitió que el programa pudiese ser sostenible durante cada año de ejecución. A manera de ejemplo, cuando el PEC inició, la Fundación de Universidad de Costa Rica (Fundación UCR) solo cobraba $10 \%$ sobre los ingresos totales por parte de por concepto de manejo de los fondos. Durante aquel periodo, este cobro o retención sobre los ingresos brutos subió a 20\%: 5\% para la Fundación UCR y $15 \%$ para un Fondo de Apoyo Institucional creado por la UCR. La innovación fue de análisis de costos y precios para lograr darle sostenibilidad financiera. Las innovaciones radicales no fueron aplicadas y no hubo mejoras tecnológicas ni en el modelo de servicios empleado.

MN1. La conciencia es excluida del diseño formal de la organización y su guía es gerencial

i. Al usar un MN en el PEC de la EM, se limitó mucho el número y tipo de actividades. El programa apenas pudo utilizar una mínima parte del potencial de conocimiento de la EM para generar eventos de EC y por su estructura tuvo poca comunicación con todos los departamentos de la EM. Los profesores de la EM tampoco lograron visualizar la relevancia de PEC durante su operación; incluso, en datos no tabulados o documentados, se consideraba que era una labor poco vinculante para los docentes.
MN2. La organización es simple y está compuesta por trabajadores que siguen procedimientos oficiales. Busca que sea predecible

i. Como se observa en la figura 2 , fue un modelo rígido y sencillo que no brindó posibilidades de responder o reaccionar ante demandas externas o nuevas. En este modelo piramidal, no existió vinculación con centros de investigación, con gremios (colegios profesionales) ni empresas privadas; tampoco existió coordinación o comunicación estrecha con los enlaces administrativos de planta de la EM.

ii. En su acción, este PEC era predecible. Se daba la instrucción de ofertar un curso y se le ofrecía el apoyo al docente contratado y se procedía con el trámite de logística y ejecución de la actividad. Se ejecutaba el curso y se redactaba un informe del curso, el cual se enviaba a la dirección para su aval.

MN3. Los trabajadores pasivos son ignorados y trabajan de forma independiente

i. Este fue uno de los rasgos newtonianos que se presentó de manera predominante. El coordinador, en este esquema o modelo, aunque tuvo la posición de trabajar directamente bajo la dirección del director de la EM, la organización no le facilitó la comunicación ni la coordinación con los departamentos docentes y administrativos, así como cualquier laboratorio o centro de investigación adscrito a la Escuela. Los cursos o las ideas de nuevos cursos surgían por impulso del coordinador o director y estos buscaban a los posibles docentes que desearan colaborar. En algunos casos, el director recibía alguna solicitud de un nuevo curso y esta era canalizada a la coordinación del PEC-EM.

ii. Los aspectos negativos de este modelo newtoniano es que el coordinador, al no estar vinculado con los departamentos docentes, no tenía forma de involucrarse de forma efectiva con todo el cuerpo docente; además, no existió 
interacción directa con la parte administrativa y hubo un recargo en aspectos administrativos, financieros y de mercadeo para el coordinador del PEC. El coordinador solamente tenía capacidad de nombrar estudiantes bajo modalidad auxiliar para apoyar los cursos. Otro efecto era la carencia de cualquier capital o recurso financiero para apoyar previamente los eventos pues todo curso debió organizarse con ingresos propios y tener excedentes para lograr ser sostenible en el tiempo. El programa operaba de forma independiente o aislado dentro de la EM, lo que se puede visualizar en la figura 2.

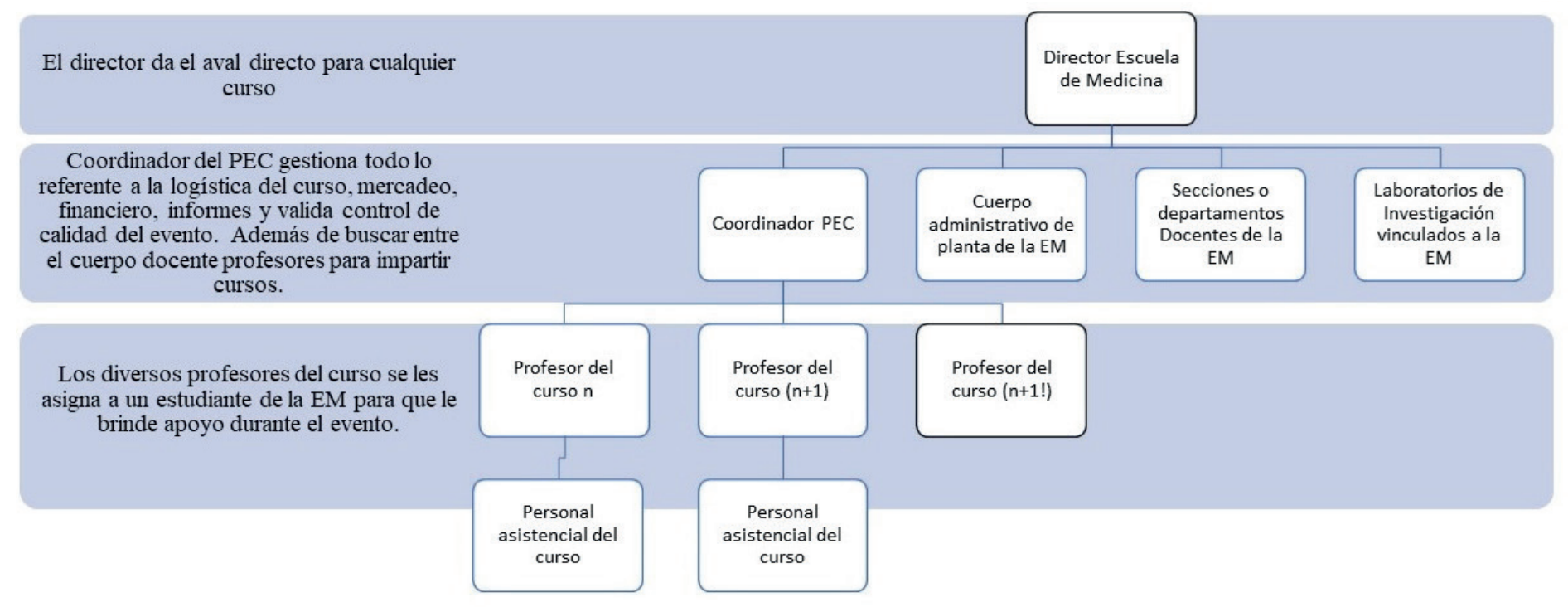

Figura 2. Esquema newtoniano del PEC de la Escuela de Medicina de la Universidad de Costa Rica ejecutado entre el 2000 y el 2003. Fuente. Campos- Rudin (2018).

MN4. La administración no está consciente de que están en una organización rígida y reactiva

Al realizar el análisis del coordinador del PEC de la EM, este siguió los parámetros establecidos y actuó de la manera que pudo reaccionar ante los eventos o actividades; no existió una estructura que pudiese ser proactiva o flexible; la meta era reaccionar frente a los indicadores propuestos y ejecutar un número de cursos o actividades por año.

Un aspecto no planificado en la metodología para elPEC dela EMfuela aplicación delelemento newtoniano MN7. La estructura rígida de la organización lleva a un proceso autodestructivo. El programa del EM concluyó sus operaciones en el 2004 (al año siguiente de cumplir los cuatros años de operación) y el coordinador fue ubicado como docente en uno de los departamentos de la EM. El conocimiento o las experiencias no se transmitieron a ningún otro funcionario, por lo cual el esfuerzo, la experiencia y los conocimientos desarrollados durante aquellos años de operación se extinguieron.

\section{PECACIBI y el MC empleado}

La EB tiene diversos grupos de especialistas en varias secciones, donde se imparten temas 
relacionados con ecología terrestre, ecología marina, zoología, botánica, micología, biotecnología, genética humana, pesquería, manejo de recursos naturales, microbiología, fitología y muchas otras áreas. Si se analiza ante la gran diversidad de temas, es imposible que se genere una masa crítica de especialistas que puedan ofertar de forma consistente cursos en una temática o temáticas particulares, y en todas las áreas se estaban generando conocimientos. En la concepción del PEC, se pensó en uno flexible y que respondiera de forma adecuada a las demandas de conocimiento de los profesionales en ciencias biológicas y la sociedad como un todo; pero, también, en cómo brindar el conocimiento de la EB hacia la sociedad.

El proceso de innovación consistió en tratar de resolver tales necesidades en un MC (figura 3). En este modelo no se presenta una estructura piramidal rígida, sino un modelo en que los diversos actores interactúan de forma activa, flexible, empoderada y con mejora continua al poder comunicarse fácilmente entre todos, además de que se incluyeron algunos rasgos newtonianos. De seguido, se describen los resultados obtenidos según el modelo cuántico.

MC1. La organización es conducida de forma flexible. Hay imaginación y experimentación

i. Se involucró a estudiantes de posgrado para que, junto con los profesores de la EB en forma conjunta, desarrollaran cursos. Se logró que algunos cursos se ejecutaran en sitios alejados de la sede central.

ii. Se gestó una alianza en temas de EC con el Colegio de Biólogos, para así acercar al gremio profesional a la Universidad.

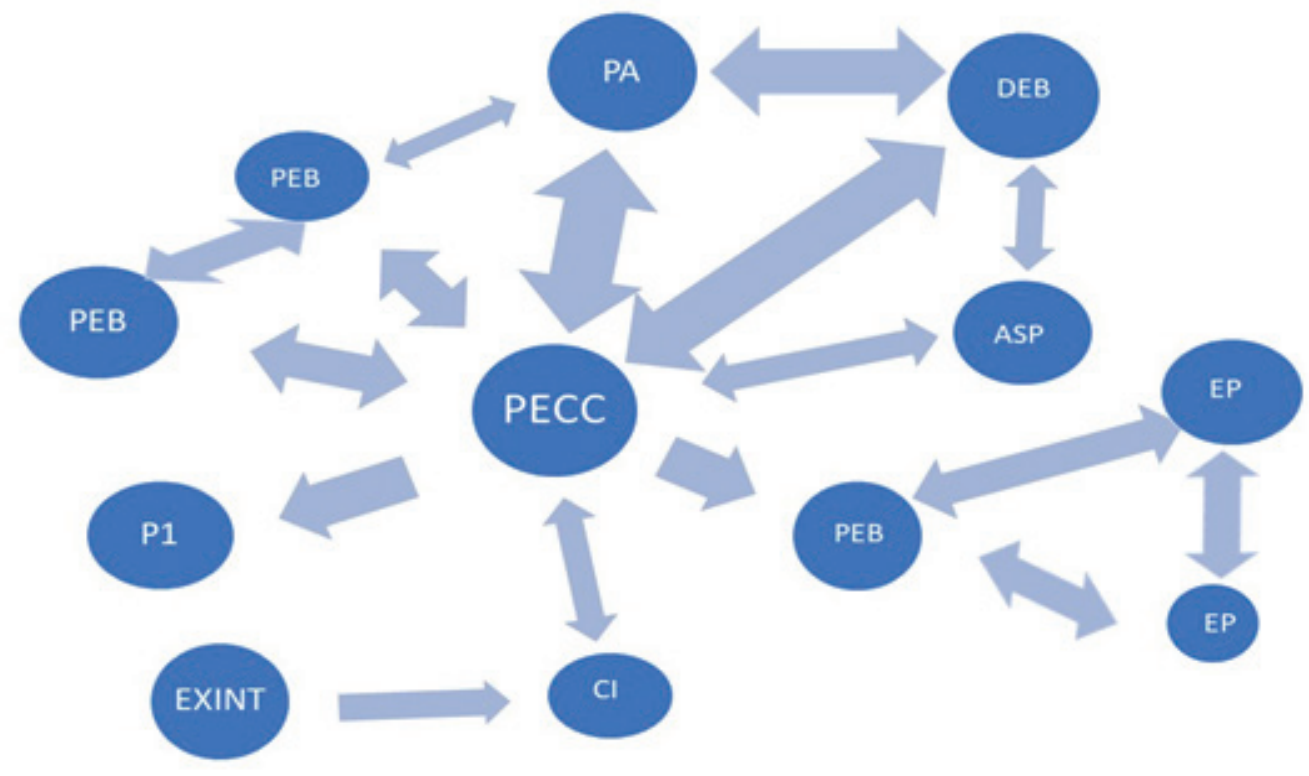

Figura 3. Modelo cuántico de organización implementado en PECACIBI en la Escuela de Biología de la Universidad de Costa Rica (2010-2013). Fuente. Campos- Rudin (2018). 
iii. Se potenció la flexibilidad en los cursos, ajustándolos a horas que fuesen viables para los docentes y atractivas para el público.

iv. Se ofreció capacitaciones a empresas y otras instituciones del sector público.

v. Se introdujoel concepto de que un PEC puede tener asociado o vinculado otros proyectos de acción social, los cuales interactúan con este cuando se gestan cursos o capacitaciones.

MC2. La organización es compleja, pero los participantes están activamente incluidos dentro del diseño de la organización (no es predecible).

i. Se motivo con asesoría del coordinador a los profesores de la EB para que desarrollaran iniciativas de EC y además invitaran a profesores de universidades extranjeras a dar cursos. Dichos cursos, en algunos casos, fueron bimodales (se ofertó a estudiantes de la EB para créditos y para profesionales egresados como un certificado de participación o aprovechamiento (esta fue una innovación sobre el proceso del programa). Los actores del PEC: el director de la Escuela, el coordinador del PEC, los docentes y el personal administrativo cooperaban de forma activa para cada evento; se buscaba la comunicación y cooperación de las partes antes que dictaminar directrices inapelables. ii. Se trabajó con centros de investigación, los cuales invitaban a investigadores del extranjero y el PECACIBI facilitó apoyo logístico. Mediante tal esquema, se lograba potenciar los recursos universitarios. En el centro de investigación, el profesor invitado realizaba los procesos de investigación y en el PEC daba acción social en forma de docencia. Gracias a este modelo la Vicerrectoría de Investigación (VI) y la Oficina de Asuntos Internacionales y Cooperación Externa (OAICE) de la UCR propiciaron la EC al facilitar la llegada de estos profesores desde el extranjero; pero no se trató de un evento programado en la metodología, sino que surgió en la operación del programa, lo cual a la vez demostró que el modelo cuántico no puede predecir todos los escenarios. iii. La comunicación entre la dirección, el PECACIBI y la planta administrativa era fluida y se colaboraba de forma equitativa.

MC6. Fuerte empoderamiento de los participantes

i. Se gestaron procesos de empoderamiento para los actores de los cursos. El coordinador del PEC asesoraba en la logística y puesta en marcha de los cursos, pero el cuerpo docente determinó el programa de los cursos. Pocos cursos fueron dirigidos por el coordinador del PEC de inicio a fin, y el director propuso cursos en pocas oportunidades.

ii. Se desarrollaron foros, coloquios o conferencias, actividades para las que PECACIBI únicamente facilitó el medio para que se inscribieran. Sin embargo, todo el proceso era gestado y desarrollado por los profesores (con muy poca participación del coordinador del PEC, salvo apoyo logístico).

MC7. Una organización flexible que se transforma de manera continua (eterna)

PECACIBI, en su formato de acción flexible, logró adaptarse y mejorar acciones al recibir retroalimentación de los profesores participantes y de los miembros de la comisión de acción social, que analizaban las propuestas al inicio y luego los informes finales. Se transformó cuando superados los primeros cuatro años de operaciones se incluyó un nuevo colaborador directo para PECACIBI, y la coordinación en los años siguientes fue compartida. Este programa continuó operando, incluso luego de que el coordinador inicial ya no estuvo vinculado (datos no presentados pues corresponden al 2017). Pero sí quedó demostrado que la organización fue capaz de evolucionar y no desaparecer. 
Este PEC al igual que cualquier otro que opere dentro del UCR debe seguir parámetros y lineamientos para operar, por ello se dio la aplicación de algunas tendencias newtonianas. La aplicación del MN2. La organización es simple y está compuesta por trabajadores que siguen procedimientos oficiales. Se busca que sea predecible; y del MN5. Controles externos a los trabajadores, fueron desarrollados para cumplir con los parámetros de evaluación y control establecidos por la UCR. En forma breve tenían que preparar reportes, informes financieros, estimación del impacto social por el número de participantes, cantidad de cursos ofrecidos, evaluaciones de la calidad del curso y presentación de informes finales, como parte de las acciones estilo newtoniano que debieron seguirse. Pero estas acciones nunca restaron el predominio de los MC1, MC2, MC6 y MC7.

En su proceso de innovación, se logróimplantar un proceso innovador semi-radical al modificar su modelo de servicios. El MC permitió realizar sustanciales mejoras el PEC, como ha sido demostrado y experimentar opciones diferentes durante su gestión. Otros tipos de innovaciones radicales usuales en PEC pueden ser acoplar mejoras tecnológicas como e-learning, módulos educativos a distancia o virtuales. Empero, estas no fueron implementadas como parte de la metodología de PECACIBI y tampoco se realizaron experiencias piloto durante el periodo analizado de este estudio. En años posteriores (datos no suministrados), se buscó implementar mejoras tecnológicas e impulsar una innovación radical, al aplicar por fondos concursables (CONARE), pero no se logró que el proyecto fuera avalado.

Comparación de resultados obtenidos por ambos PEC en sus primeros cuatro años de operación

En los PEC, una forma básica de medir su incidencia es la cantidad de eventos de capacitación ofertada y ejecutada, variedad temática, población meta (número de asistentes, profesión, lugar de residencia y otras variables sociodemográficas), calidad del evento (por ejemplo, realizaruna encuestadesalida)eingresos obtenidos para sostener estas actividades. Al comparar los resultados de ambos PEC a partir de estos criterios, se puede considerar que ambos son similares en cuanto al número de eventos y que, durante su ejecución, fueron sostenibles desde el punto de vista económico (tabla 2). Pero, adicionalmente, se lograron obtener otros resultados diferenciadores que no son usualmente evaluados, como inserción de profesores de otros países y presencia de estudiantes de posgrado e interacción de docentes de la EB, oferta de servicios externos a empresas y trabajo del estado en temas requeridos y su continuidad en el tiempo. Todo fue detallado en los MC empleados.

El coordinador del PEC en PECACIBI, dado el $\mathrm{MC}$, se pudo concentrar más en procesos motivadores, inductivos, gestores de actividades y buscar formatos de innovación en los tipos de cursos. Esto se logró al no recaer sobre su gestión aspectos financieros y administrativos (los cuales fueron apoyados directamente por el personal administrativo de la EB). Otro aspecto relevante es que impulsó al grupo de docentes en cada curso para que fueran los responsables de la parte académica (programa del curso). No obstante, sí hubo eventos gestados desde la dirección y la coordinación, y el programa si fue organizado entre PECACIBI y los docentes.

La relación ingresos y gastos fue positiva, lo cual brindó sostenibilidad en el corto plazo, pero no aseguró ser sostenible y crecer en el largo plazo. Cualquier organización que no facilita el proceso de transición o entrenamiento de nuevos liderazgos no puede evolucionar. PECACIBI fue sostenible y creció (datos no suministrados) hasta el 2017, mientras que el PEC-EM no pudo 
continuar después del 2014 y no trasmitió conocimientos a otros potenciales actores.

La no continuidad del PEC-EM demostró que un $\mathrm{MN}$ estricto lleva a este proceso mencionado en el MN7 (autodestrucción). Esto soporta la concepción de un modelo mecánico newtoniano descrito en la teoría, que busca obtener un número de resultados, y cuando estos se logran la organización, de forma predecible, no evoluciona y decae. El PEC-EB tuvo la posibilidad de potenciar un MC y adoptó algunos elementos requeridos del $\mathrm{MN}$, pero con predominio cuántico, lo cual mostró que además de dar cursos, fue posible innovar y experimentar nuevos formatos, recibir retroalimentación y evolucionar. Además, su continuidad en años posteriores logró demostrar que este $\mathrm{MC}$ brinda los elementos para unir la EC, como un proceso de puede evolucionar con los seres humanos en sus necesidades de conocimiento en la cuarta revolución industrial. Las PEC deben ser diseñados para que perduren en el tiempo pues deben ser concordantes con el concepto de EC que expone que debe ser a lo largo de la vida de las personas el diseñar programas que puedan evolucionar.

Tabla 2

Análisis comparativo de ambos PEC en su gestión y organización

PEC-EM

PECACIBI

Cantidad de eventos No más de 10 (inicio sin antecedentes

por año de un PEC o proyecto de EC).

Tipo de actividades Cursos y colaboración en congresos.

Poblaciónbeneficiaria Médicos. En pocos eventos se brindaba capacitaciones a otras disciplinas.

Impacto financiero Programa sostenible, pero con pocos excedentes para ayudar en la gestión de la Escuela.

Personal Docente Pocos profesores se involucraron y fueron, en su gran mayoría, profesores de la EM.

Incentivos a docentes Se brindó remuneración económica.

Se brindó remuneración económica y otros incentivos como apoyos para investigación.

Vinculaciones Vinculación puntual con la Facultad de Farmacia.

Mínimo 10 (tuvo un antecedente o proyecto previo conocido como Biología para Todos).

Múltiples cursos, asesoría, foros, charlas, simposios y congresos.

Enfoque diverso: biólogos, estudiantes de pre y posgrado, público en general y profesionales de áreas afines a la biología.

Programa sostenible y brindó apoyo a la EB en compra de materiales y equipos.

Diversidad en cuanto al número de profesores, participaron profesores de universidades extranjeras e incluso estudiantes avanzados de posgrado.

Vinculación con Colegio de Biólogos, Centros de Investigación. y otros proyectos de acción social.

Continúa... 
Trabajo del coordinador y su relación con el entorno

Modelo y tipo de proceso innovador

Sostenibilidad a largo plazo
Funciones administrativas, control de ingresos / egresos, poca vinculación o comunicación con la sección administrativa y departamentos docentes de la EM.

30 horas semanas de carga asignada.

Newtoniana y de estructura rígida. Innovaciones incrementales para sostener el PEC.

No logró continuar vigente, al no contar con una alternativa para transformarse o evolucionar.
Alto nivel de comunicación y de apoyo con la sección administrativa.

Vinculaciónconelcuerpodocentey estudiantil. 10 a 15 horas de carga semanal asignadas.

Cuántica y de estructura flexible. Innovación semi-radical, basado en mejoras en el modelo de servicos de la organización

Hasta diciembre de 2017, se hallaba vigente, logró crecer y sostenerse con 8 años de funcionamiento ininterrumpido.

Fuente. Campos- Rudin (2018)

\section{Discusión}

El nuevo paradigma educativo en occidente está basado en un proceso mecánico de tipo industrial, en el cual existen tres estrategias: la estandarización, la privatizaciónyla competencia. De estos tres, el tema de la estandarización justifica el presente estudio: dicha estrategia establece que los programas de estudio son únicos y rígidos, preestablecidos, basados es asignaturas separadas disciplinariamente con escaso margen de flexibilidad para las escuelas y docentes (Severin, 2017). La estrategia de estandarización muestra un MN típico; y un modelo con intromisión cuántica es una nueva estrategia que pueden innovar nuevos modelos educativos (Giraldo, 2017).

Para lograr permanecer vigentes antes las demandas crecientes y cambiantes de educación en nuestras sociedades, los PEC requieren gestar procesos de innovación dentro de sus organizaciones y en las UAI deben ser capaces de atraer a los docentes investigadores a participar activamente. En algunos casos, esta innovación puede ocurrir al introducir agresivamente tecnologías de información y sistemas de aprendizaje en línea dentro de la organización (Lu, Chang, Yan, \& Lin, 2016) (tipo radical), pero estos según Severin (2017) son insuficientes. En estos formatos, el desarrollo del e-learning es un tipo de innovación alterna a la clase magistral presencial (Pehlivanova \& Ducheva, 2010). Solamente aplicar innovaciones tecnológicas, brindar plazas docentes, dar incentivos salariales o menciones especiales a los docentes participantes serán acciones insuficientes para que los PEC logren vincularse efectivamente a la era de la cuarta revolución industrial, puesto que muchas de estas mejoras son medios para facilitar procesos educativos o de gestión; pero no asegura que la organización crezca y sea sostenible en el tiempo.

En nuestros dos casos, el ofrecimiento de pagos salariales adicionales por dar cursos no fue un evento motivador y es evidente que no incentivó la permanencia del PEC-EM en el tiempo. En PECACIBI no se logró realizar innovaciones tecnológicas; pero en el modelo de servicios sí se logró innovar con el MC y experimentar durante toda su operación. Ejemplos de estos procesos fueron las poblaciones atendidas, lugares donde se brindó capacitación, cursos bimodales, vinculación con otros proyectos de acción social, atención de servicios de empresas o sector público y coordinación otras dependencias del UCR para traer profesores del exterior. 
Un error adicional que se cometió en la EM fue la no vinculación de los PEC con la cotidianidad de las labores o necesidades de la sociedad en conocimiento. Se pueden encontrar procesos de EC en que los trabajadores o los públicos meta son poco considerados (Chiodelli \& Lenise, 2007). Un PEC sin vinculación con colegios profesionales, sectores productivos o sociedad no podrá tener una incidencia sostenible en el entorno y mediante un $\mathrm{MN}$ es más difícil movilizar la organización hacia nuevos entornos. Se razona, nuevamente, que el modelo de PECACIBI permitió no caer en este error.

López (2013) describe una serie de modelos operativos de los PEC y se observan estructuras de tipo newtonianas, que siguen un modelo clásico del mundo occidental: se gestionan estructuras piramidales, con gran orden jerárquico y funcionalmente efectivas en su momento, al orientarse a metas y objetivos concretos de las organizaciones (figuras 1 y 2). Pero tales organizaciones se convierten en uno de los principales obstáculos para el desarrollo y el progreso en nuestro entorno (Yavas \& Polat, 2015). Este razonamiento se basa en el modelo mecanicista newtoniano, cuyas estructuras rígidas no facilitan la interdisciplinaridad.

Los MC descritos por Kilmann (2011), por el contrario, promueven la interacción que demandan la cuarta revolución industrial, donde debe privar la fusión de lo biológico con lo tecnológico (Schwab, 2016). El modelo cuántico genera los cuadros operativos flexibles, dinámicos e innovadores que se requieren (figuras 1 y 3) para promover un desarrollo sostenible en el largo plazo en los PEC.

Finlandia es un país que ha sido premiado y reconocido a nivel mundial por su sistema educativo formal; de manera congruente, el sistema finlandés de educación permanente está ligado al sistema educativo finlandés por los principios de equidad, alto nivel educativo y flexibilidad. En referencia a este punto de flexibilidad, el sistema finlandés evitó llegar a "calles sin salida o ciegas", donde las personas pueden continuar su educación o complementarla en cualquier nivel de su sistema educativo (Niemi \& Isopahkala-Bouret, 2012). Aunque no se busca determinar si en el sistema educativo en EC de Finlandia predomina el MN o el MC, lo que sí es destacable es su sistema flexible a la persona, buscando equidad en la migración de conocimientos; así pues, los PEC de las unidades académicas que deseen ser exitosos y equitativos deben permitir el ingreso o la participación de personas en cualquier nivel de etapa educativa.

PECACIBI, por ejemplo, en este punto, dio cursos abiertos a la comunidad; incluso, se atendió a población con síndrome de Down. Otro concepto para la EC es que debe llegar donde sea requerida y lograr cumplir esta demanda (Cuitún, 2002). Nuevamente, la flexibilidad es importante y este concepto permitió que en PECACIBI, al usar el MN1, se pudiese ejecutar capacitaciones fuera de la UCR como el caso de la Isla de Chira; pero esto no fue contemplado en el PEC-EM, donde el lugar de operaciones fue siempre la EM.

En un modelo cuántico, también es viable establecer alianzas estratégicas como con diversidad de unidades académicas, centros de investigación y colegios profesionales; en PECACIBI fue posible y se desarrollaron modelos tipo Win-Win que facilitan optimizar los recursos y potenciar el intercambio de conocimientos (Velasco, 2015). Si una organización como un PEC desea ser innovadora, debe aprender a convivir con más preguntas que respuestas y debe sentirse cómoda dentro este entorno (Larrea, 2008). El MC1 permitió que PECACIBI pudiese ser una organización innovadora.

El perfil del coordinador en cuanto a su papel de líder, al buscar innovar de un programa a 
otro, debe ser analizado en ambos modelos. En el marco de un concepto newtoniano, el líder se perfila más como un directivo o gerente, busca predecir, dar resultados y controlar todos los eventos; mientras que en un modelo cuántico, el liderazgo toma una posición relevante (Zohar, 2016).

En el modelo cuántico, la visión es de una forma más sencilla: se busca inducir, experimentar y guiar con mucha consciencia por el camino natural de la organización, permitiéndole al líder participar en el desarrollo de la organización, pero sin buscar controlarla. Se busca impulsar el crecimiento libre de la organización (Shelton \& Darling, 2001) y se puede especular que la aplicación de liderazgo con uso de la inteligencia emocional puede ser un impulsador de un liderazgo cuántico, ya que en su esencia los estilos tipo coach, visionario y conciliador propuestos por Goleman (2011) se introducen bien dentro de este formato de líder. Dentro del MC, se plantea, además, que todos podemos ser líderes, y la incidencia del liderazgo dependerá de las interacciones con sus colaboradores; donde se plantea de forma clara los líderes son guías y no jueces (Ercetin \& Kamacr, 2008).

En el PEC-EM, el liderazgo fue newtoniano, enfocado en lograr metas en cuanto a número de cursos y obtener sostenibilidad financiera. Asimismo, se determinaron tipos de cursos y acciones de manera estructural, y el coordinador controló y dominó toda la organización. En su papel gestado en la EB, el perfil fue de tipo cuántico; no se buscó imponer cursos o temas, sino más bien incentivar a los profesores para que estos propusieran. Igualmente, se auspició cursos bimodales (presencia de estudiantes regulares de EB y egresados), se buscó diversidad y se habilitó el acceso a población no profesional a los cursos. La EB, como una organización, pudo utilizar y fluir con el programa hasta lograr metas exitosas compartidas.

Todo proceso innovador debe requerir subprocesos de gestión para su adecuado monitoreo y seguimiento. $\mathrm{Y}$ aunque en el MC se puede inducir a pensar que el control o la supervisión de los eventos es complejo debido al empoderamiento y a la flexibilidad de la organización, se pudieron aplicar los mismos indicadores de control en PECACIBI como en el PEC-EM. Incluso, el PEC de la EB tuvo más controles, al tener que rendir informes no solamente al director, sino a una comisión de acción social de la EB. La inclusión de algunos MN1 dentro delagestión deun MC predominante logró establecer un balance de innovación y control necesario para asegurar un crecimiento y la transformación continua de PECACIBI.

\section{Conclusiones}

El PEC-EM, desde su estructura newtoniana, pudo cumplir sus objetivos de brindar una opción para capacitar a los profesionales egresados de la EM; pero no logró unificar o despertar en su entorno un interés general de los docentes para involucrarse activamente dentro del PEC (salvo algunas excepciones). Su estructura enfocada en resultados no le permitió llegar a potenciar su crecimiento, presentó un proceso de innovación limitada y no pudo evolucionar en el tiempo.

Por el contrario, PECACIBI pudo innovar de forma semi-radical con un MC y adoptó algunas posturas Newtonianas, y logró posicionarse en la EB como una organización interna exitosa entre el 2010 y el 2013. Al aplicar estrategias orientadas hacia flexibilidad, experimentación, empoderamiento de actores, retroalimentación continua y búsqueda de una eterna transformación, logró desarrollar actividades de forma sostenible en el tiempo y no autodestruirse. 
Posteriormente a los rangos de fecha descritos en este estudio, PECACIBI logró continuar transformándose hasta el momento de estudio de esta publicación (2017) y amplió un nódulo de acciones, como organización directa de simposios y congresos (datos no suministrados) y participación en concursos con fondos concursables, interacción con otras universidades públicas de Costa Rica. Otro efecto que mostró su fuerte evolución fue la transición de coordinación a nuevas personas después del 2014.

En síntesis, los PEC, así como cualquier organización que desee perdurar, crecer y ser exitosos a lo largo del tiempo, deben operar de una forma cuántica y con personal dispuesto a afrontar nuevos retos (Kilmann, O'Hara, \& Strauss, 2010).

\section{Agradecimientos}

Es imposible agradecer a todos los profesores $\mathrm{y}$ administrativos que colaboraron en ambos PEC. Mencionaré en la Escuela de Medicina a Jollyanna Malavassi y a Silvia Mata Roldán por su apoyo fuerte al PEC-EM. En la Escuela de Biología, el apoyo del personal administrativo y docente fue vital. A la Dra. Hilda Sancho, como directora de la Escuela de Medicina, y al Dr. Gustavo Gutiérrez, como director de la Escuela de Biología, por el impulso de los PEC. A los integrantes de la comisión de acción social de la Escuela de Biología por sus sugerencias y revisiones para implementar un mejor PEC.

\section{Referencias}

Anderson, W., Banerjee, U., Elgin, R., \& Handelsmann, , J. (2011). Changing the culture of science education at research universities. Science, 331, 152-153.

Campos-Rudin, M. (2017). Gestión y desarrollo de un programa de educación continua por la Escuela de Biología de la Universidad de Costa Rica. Revista Gestión de la Educación, 7, 115-132. Obtenido de http://revistas. ucr.ac.cr/index.php/gestedu

Chiodelli, N., \& Lenise, M. (2007). Educación continua en el trabajo: nuevos desafíos para los profesionales de enfermería. Investigación y Educación en Enfermería, 25, 100-105.

Cuitún, M. (2002). Estrategia para mejorar la calidad de la educación continua. Revista de la Universidad Autónoma de Yucatán, 222, 14-24.

Dávila, A. (2008). ¿Que se puede hacer para diseñar organizaciones innovadoras? En A. Dávila, Innovar o Desaparecer (pág. 160pp). Barcelona: Ediciones Deusto.

de Bes, T., \& Kotler, F. (2011). Innovar para ganar. El modelo ABCDEF. Barcelona, España: Ediciones Urano S.A.

Ercetin, S., \& Kamacr, M. (2008). Quatum leadership paradigm. World Applied Sciences Journal, 3, 865-868.

Giraldo, J. (2017). Intromisiones cúanticas en educación. Eduación y Ciudad, 32, 65-74.

Goleman, D. (2011). Liderazgo, el poder de la inteligencia emocional (1 edición ed.). (C. Mayor, Trad.) Barecelona, España: Ediciones B, S.A.

Jones, R. (2017). Active learning in nurse education: using quantum learning and campbellteaching to improve retention. The International Journal of Pedagogy and Curriculum, 24, 29-40.

Kilmann, R. (2011). Quantum Organizations, A new paridigm for achcieving organizational success and personal meaning. California, USA: Orginizational Design Consultants.

Kilmann, R., O'Hara, L., \& Strauss, J. (2010). Developing and validating a quantitave measure of organizational courage. Journal of Bussiness Phsycology, 25, 15-23. 
Laal, M., Laal, A., \& Aliramaei, A. (2014). Continuing education; lifelong learning. 5 th world conference on educational sciences-WCES 2013. Procedia-Social and Behavioral Sciences, 116, 4052-4056.

Larrea, J. (2008). El lenguaje de la innovación: poética y práctica. En A. Dávila, Innovar o desaparecer (pág. 160p). Barcelona: Ediciones Deusto.

López, M. (2013). Modelos universitarios de gestión de la educación continua. RECLA: retos y claves de la educación continua, 1, 51-62.

Lu, H., Chang, K., Yan, M., \& Lin, P. (2016). Organizatinal innovation for continuing education in higher educationi in Taiwan: The case of the Chinese Culture University. International Journal of Continuing Education and Lifelong Learning, 8, 128-144.

Marques, J. (2014). Closed versus open innovation: evolution or combination? International Journal of Business and Management, 9, 196-203.

Niemi, H., \& Isopahkala-Bouret, U. (2012). Lifelong learning in Finnish society: an analisys of national policy education. International Journal of Continuing Education and Life Long Learning, 5, 43-63.

Pehlivanova, M., \& Ducheva, Z. (2010). Role of flexible educational models for lifelong learning. Trakia Journal of Sciences, 8 suppl (3), 348-354.

Schwab, K. (2016). La cuarta revolución industrial. España: Penguim Randon House Group Editorial España. Edición Kindle.

Severin, E. (2017). Un nuevo paradigma educativo. Educación y Ciudad, 32, 75-82.

Shelton, C., \& Darling, J. (2001). The quantum skills model in management: a new paradigm to enhance effective leadership. Leadership \& Organizational Devolopment Journal, 22, 264-273.

Tuschling, A., \& Engemann, C. (2006). From education to lifelong learning: the emerging regime of learning in the European Union. Educational Philosophy and Theory, 38, 451-469.

Velasco, J. (2015). Alianzas estratégicas en la universidad: un modelo win-win. Retos y Claves de la Educación Continua, 3, 48-55.

Velazco, A. (2011). La educación continua como instrumento de desarrollo en el siglo XXI. Tendencias, Revista de la UBP, 5, 2-5.

Yavas, T., \& Polat, M. (2015). Organizational development and quantum organizations. International Journal of Social Sciences \& Education, 5, 570-579.

Zohar, D. (2016). The Quantum Leader: a revolution in bussiness thinking and practice. New York: Prometheus Books.

Recibido: 27 de enero de 2018

Aceptado: 09 de mayo de 2018 
